\title{
Ad Honorem Claire Voisin
}

Arnaud Beauville, Guest Editor

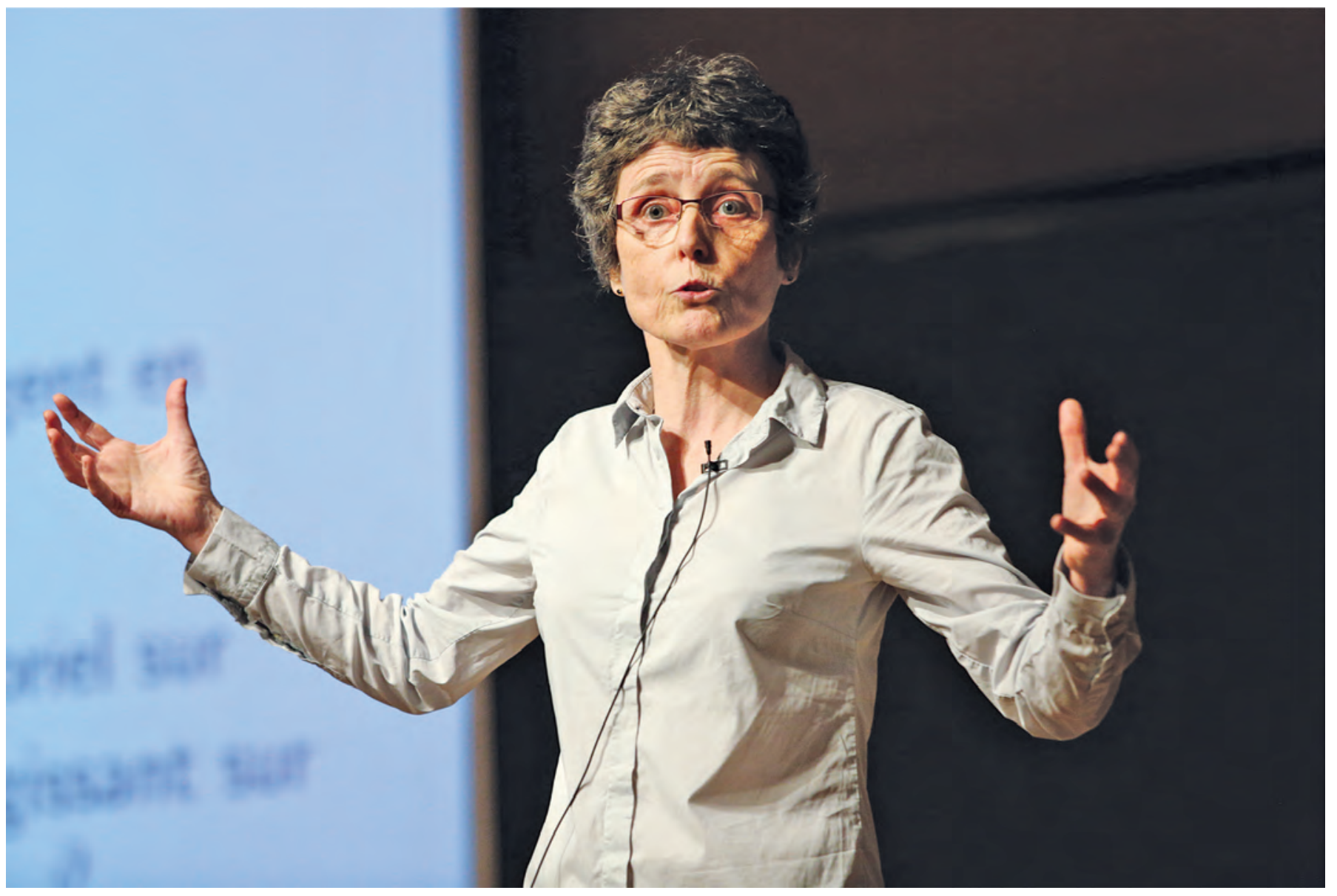

For permission to reprint this article, please contact:

reprint-permission@ams.org.

DOI: http://dx.doi.org/10.1090/noti1665 


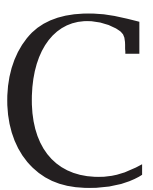

laire Voisin is a world leader in algebraic geometry. After a PhD thesis under Arnaud Beauville at Orsay, she entered CNRS, where she stayed until 2016, when she became professor at the prestigious Collège de France.

The dominant theme of her work is Hodge theory, in particular, its application to concrete classical problems. She solved the Kodaira problem by constructing a compact Kähler manifold that cannot be obtained by deforming a projective manifold. In Noether-Lefschetz theory she proved existence results for subvarieties of a given projective variety. She made important advances on the integral Hodge conjecture, leading to a breakthrough on the Lüroth problem on rationality questions. She proved the Green conjecture for a general curve and deep results on hyperkähler manifolds.

Such fundamental results, which are detailed in the five contributions below, have been rewarded by numerous awards and distinctions. Some of the most prestigious are the Heinz Hopf Prize (2015), the gold medal of CNRS (2016), and the Shaw Prize (2017). She gave a plenary address at the Hyderabad ICM (2010). She is a member of the French Académie des Sciences and of half a dozen foreign academies.

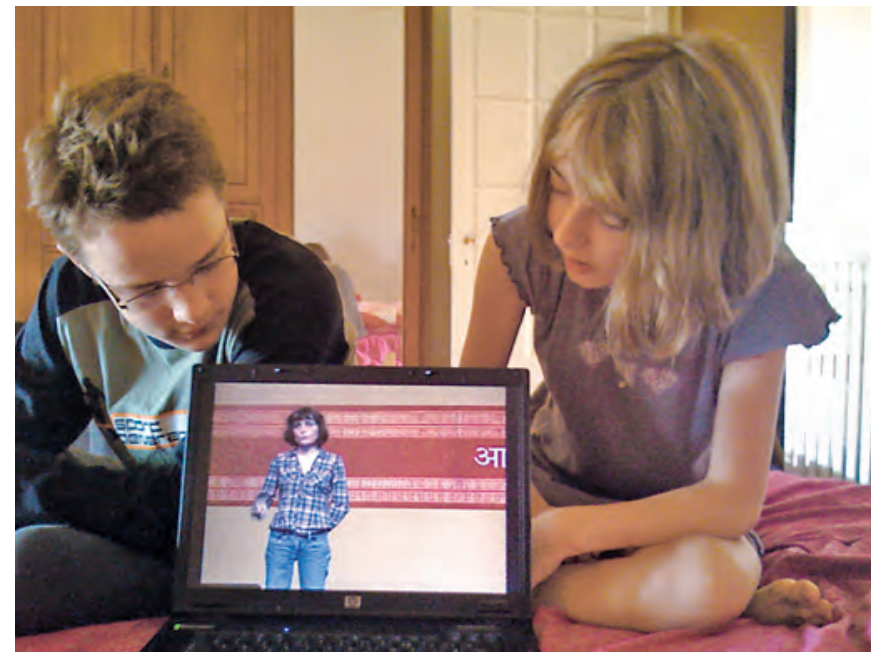

Voisin's children watch her ICM plenary. Voisin writes, "It was early in the morning in France due to the time difference. They were so stressed that they could not watch very long."

\section{Ekaterina Amerik}

\section{On Claire Voisin as a Role Model for My Generation}

Upon the fall of the USSR, along with many other Russian graduates who wanted to go on with scientific research, I left Russia. One of the first things my thesis advisor, A. Van de Ven, of Leiden University, told me was, "At some point you should go to Paris. To Claire Voisin."

The prospect of going to Paris at some point was terrific. Naturally, I thought of Claire Voisin as a venerable professor, possibly a bit younger than Van de Ven himself, in his early sixties at the time, and probably single: the common knowledge in Russia was that those very rare women who successfully start a career in mathematical research are most likely to forget it once the children are born.

I actually saw Claire for the first time before seeing Paris-she was lecturing at a summer school in Torino, I had met before at the end of the same academic year-and was startled to find out that she was only slightly older than many students in the audience, already had three children, and was more swift and determined than any person I had met before.

The subject of the summer school was "Algebraic cycles and Hodge theory." To give the first flavor of this subject, let me mention the classical result already guessed by Max Noether, the proof of which goes back to Lefschetz: any curve on a sufficiently general hypersurface $X_{t}$ of degree at least four in $\mathbb{P}^{3}$ is a complete intersection. By the Lefschetz theorem on $(1,1)$-classes, this is implied by a cohomological version: any $(1,1)$-class $\lambda \in H^{2}\left(X_{t}, \mathbb{Z}\right)$ is the restriction of a class from $H^{2}\left(\mathbb{P}^{3}, \mathbb{Z}\right)$. Lefschetz's approach is as follows. A class surviving on a general hypersurface $X_{t}$ should be invariant by a finite index subgroup in the monodromy. On the other hand, one can show that the monodromy is a large group, in a sense as large as possible. Indeed its action on the primitive cohomology preserves the intersection form. So for small degrees, when this form is definite it has to be a finite group. Yet it turns out that whenever the intersection form is indefinite, that is, starting from degree four, any finite subgroup of the monodromy acts irreducibly.

It is clear from this discussion that the locus of "nongeneral hypersurfaces" (those carrying an extra integral $(1,1)$-class $)$ in the parameter space $\mathbb{P} H^{0}\left(\mathbb{P}^{3}, \mathcal{O}_{\mathbb{P}^{3}}(d)\right)$ is a countable union of proper subvarieties. It is often called "the Noether-Lefschetz locus." Deligne in Séminaire de la Géométrie Algébrique 7, II gives an algebraic version

Ekaterina Amerik is professor of mathematics at Université ParisSud and also a member of the Laboratory of Algebraic Geometry at HSE, Moscow. Her email address is Ekaterina. Amerik@math .u-psud.fr.
Arnaud Beauville is professor emeritus at Laboratoire J.-A. Dieudonné, Université de Nice. His email address is arnaud .beauvi11e@unice.fr. 
of Lefschetz's argument that provides some extra information on this locus, showing that an equation whose coefficients are algebraically independent over the prime field defines a hypersurface with cyclic Picard group, hence outside the Noether-Lefschetz locus.

Starting with the work of Carlson, Green, Griffiths, and Harris, people have been trying to understand this and similar loci better using the theory of infinitesimal variations of Hodge stuctures. The tangent space to the component where a primitive class $\lambda$ stays of type $(1,1)$ is given by the kernel of the multiplication by $\lambda, \cup \lambda: H^{1}\left(X_{t}, T_{X_{t}}\right) \rightarrow H_{\text {prim }}^{0,2}\left(X_{t}\right)$ (here we consider the moduli of $X_{t}$ rather than the "larger" parameter space $\mathbb{P} H^{0}\left(\mathbb{P}^{3}, \mathcal{O}_{\mathbb{P}^{3}}(d)\right)$, but the passage from one to another is standard). It turns out that the tangent space to the moduli $H^{1}\left(X_{t}, T_{X_{t}}\right)$, as well as the summands $H_{\text {prim }}^{p, q}$ of the Hodge decomposition of the primitive cohomology of a hypersurface defined by the equation $F=0$, are identified with the graded components of the Jacobi ring (the quotient of the coordinate ring by the Jacobian ideal of $F$ ). This makes it possible to translate problems on the Noether-Lefschetz and similar loci into a subtle mix of commutative algebra (Koszul-type complexes for Jacobi rings) and projective geometry. By the time we were listening to Claire's lectures, she had already shown that the only component of the Noether-Lefschetz locus having minimal possible codimension $d-3$ was the family of surfaces containing a line, ${ }^{1}$ and the next largest one, the only one of codimension $2 d-7$, was the family of surfaces containing a conic. This would seem to support a conjecture by Joe Harris that only finitely many components of the Noether-Lefschetz locus have codimension greater than the $\left({ }^{d-1}\right)$ predicted by the dimension count; however, Claire has shown that this conjecture is false. But she also went much further, applying similar techniques to higher-codimensional algebraic cycles. An algebraic cycle of codimension $k$ is an integral linear combination of $k$-codimensional subvarieties. Usually one considers the algebraic cycles modulo rational equivalence (two cycles are said to be rationally equivalent when their difference is the zero/pole divisor of a rational function on some subvariety of codimension $k-1$ ); the quotient is called Chow group. When $k=1$, this is just linear equivalence, and the Chow group is an extension of a discrete group (the image of Picard group in $H^{2}$ ) by a torus. For greater values of $k$, the Chow groups are normally much bigger: as observed by Mumford, the existence of differential forms implies that they are "infinite-dimensional" and there is no hope to parametrize them by anything remotely similar to algebraic varieties. Still one can define the Abel-Jacobi map from the cohomologically trivial part of the Chow group to a torus built from the Hodge structure. Infinitesimal techniques enabled Claire to show that for codimension-two cycles on a general hypersurface of degree at least six in $\mathbb{P}^{4}$ the image of the Abel-Jacobi map is torsion, to give a new proof of the famous result by

${ }^{1}$ This result and the subsequently cited one on the Abel-Jacobi map have been proved independently by Mark Green.
Clemens that on a general hypersurface of degree five it is not only nontorsion but even infinite-dimensional when tensored up with the rationals, and to do many other things in this direction.

Probably the most striking application of those techniques in Claire's work of the time was a result from a 1994 Math. Annalen paper about zero-cycles on surfaces. By introducing suitable infinitesimal invariants she showed that on a general surface of degree $d \geq 5$ in $\mathbb{P}^{3}$, a nontorsion zero-cycle in the jacobian of a general plane section was nontrivial in the Chow group of the surface, for $d \geq 6$ only finitely many points were rationally equivalent to a given point $p$, and for $d \geq 7$ there were no such points at all. To be honest, my attempts to understand the proof of the main result were not successful; what I was

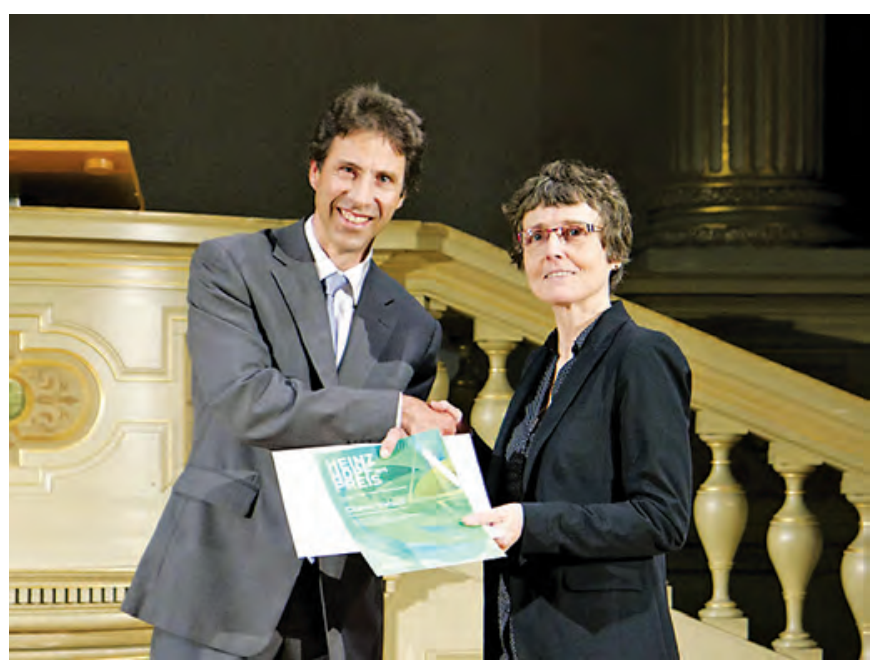

Voisin, pictured here with ETH Zürich's Peter BühImann, received the Heinz Hopf Prize in 2015.

trying to understand, by different means, was the simple implication that a general surface of degree $d \geq 5$ could not contain any rational curves and its extension to higher dimensions and to higher genera (i.e., what is the minimal geometric genus that a curve on a general hypersurface of degree $d$ can have). In fact the result on rational curves had been known before, since Clemens's work in 1986: his theorem states that a general hypersurface of degree at least $2 n-1$ in $\mathbb{P}^{n}$ does not contain rational curves. This is sharp for $n=3$.

A beautiful observation in Claire's paper published in the Journal of Differential Geometry in 1996 was that the bundle $\left.T_{\chi}(1)\right|_{X_{t}}$, where $\chi \subset \mathbb{P}^{n} \times S_{n}^{d}$ is the universal family of hypersurfaces and $X_{t}$ a general member, is generated by the global sections. This gives a similar assertion on the bundle of differential forms of suitable degree and translates into the positivity of the canonical divisor of the subvarieties. This approach allowed her to unify and sharpen several previous results: the above-mentioned one by Clemens, the generalization for higher-dimensional subvarieties by Ein, and a stronger result for divisors by Geng $\mathrm{Xu}$. Then Claire went on to study the base locus 


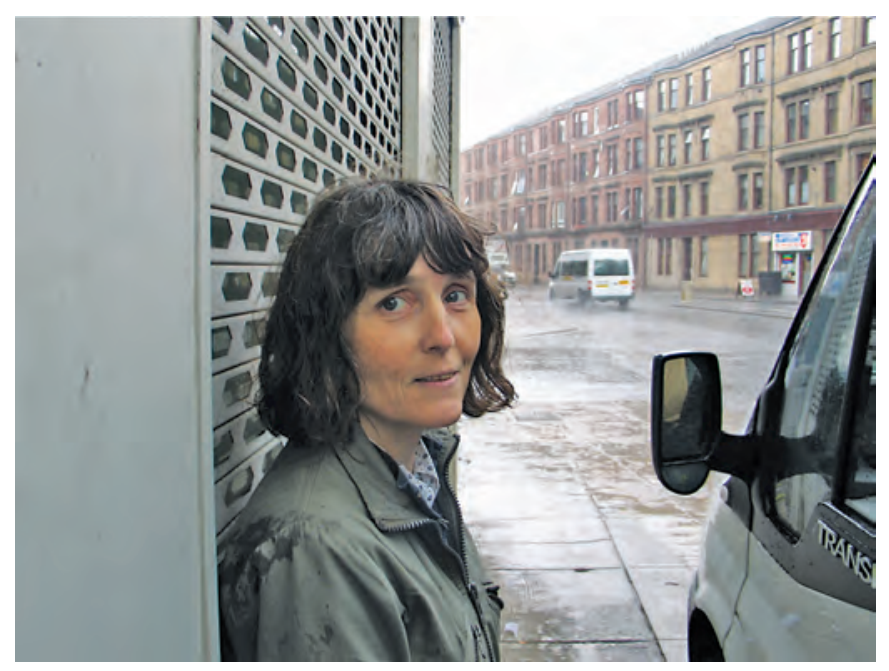

Voisin in Glasgow in 2009.

of $\left.\Lambda^{2} T_{X}(1)\right|_{X_{t}}$ in order to improve on the Clemens-Ein bound for $n \geq 4$ : the improvement stated, for instance, the absence of rational curves on a general hypersurface of degree at least $2 n-2$ in $\mathbb{P}^{n}$ (which is sharp since hypersurfaces of degree $2 n-3$ contain lines).

I read the paper with great enthusiasm as finally I could understand most of it (by the way, Claire refers to this paper as one of her less deep works). But still it was a couple of months before I realized that the argument about the base locus of $\left.\Lambda^{2} T_{\chi}(1)\right|_{X_{t}}$ could not be correct for obvious reasons: it would contradict the dimension count for subvarieties covered by lines when $\operatorname{deg}\left(X_{t}\right)$ slightly exceeds $2 n-3$. I wrote to Claire by regular mail (it was still unusual or even impossible to send anything but short messages electronically). The answer, in the form of a ten-page mathematical paper-an erratum correcting the proof to reassert the validity of the main theorem and giving some other interesting details about the base locus in question-came after just a few weeks.

For a long time I had been observing Claire's work at a distance, and then I had a very unexpected occasion to get somewhat closer. I will finish my account with the story of our collaboration.

Obvious examples show that an algebraic variety defined over a field $K$ does not necessarily have $K$-valued points. However, the points appear when one replaces $K$ by a suitable finite extension $L$. Sometimes the $L$-points are scarce, as is the case for curves of genus at least two (the famous Mordell conjecture, proved by Faltings, states that there is only a finite number); sometimes these are many. If one can choose $L$ in such a way that the set of $L$ points is not contained in any proper subvariety, one calls the algebraic variety potentially dense. Such is the case for unirational varieties or for abelian ones. One would like to characterize the potential density geometrically, and there are grounds to believe that in the smooth case it is related to the positivity properties of the canonical line bundle (i.e., the determinant of the cotangent bundle): the varieties with positive canonical bundle should not be potentially dense, whereas those with negative or zero canonical bundle should. In the negative case, examples abound, but in the zero case the known ones are not very convincing. Even for projective K3 surfaces, it is an open question whether a general one in any maximal family is potentially dense. Together with Frédéric Campana, we made an attempt to produce some new examples dynamically, in the following sense. There is a four-dimensional analogue of a K3 surface, varying in a certain maximal family, such that each member of the family is equipped with a dynamically interesting rational self-map $f$ (the construction, by the way, is again due to Claire!), and one hopes to get many new $L$-points by iteration. Indeed, if $x$ is one such point, so are $f(x), f^{2}(x)$, etc. One hopes that the iterates of a sufficiently general $x \in X(L)$ won't be contained in a proper subvariety unless there is a clear geometric reason for this: the map preserves a fibration. Around 2005, we made this idea work when the field $K$ is uncountable, but the most interesting case is that of a countable $K$-for instance, $K=\mathbb{Q}$-and then it might happen that no $\overline{\mathbb{Q}}$-point of $x$ is sufficiently general in this sense!

The reader who still recalls the Noether-Lefschetz story can make a parallel with Deligne's version of it: for surfaces whose coefficients are as transcendental as possible one can easily draw certain conclusions, whereas the surfaces with algebraic coefficients are more subtle. This subtlety has been addressed by T. Terasoma, who proved around 1985 that most surfaces defined over $\overline{\mathbb{Q}}$ still have cyclic Picard group. He proceeded by a comparison of the monodromy and the Galois action on the second cohomology of such a surface, and his success gave some clue that our problem was somehow solvable.

Still I could not figure out a solution. The distance between the classes of line bundles on a surface and orbits of points on a fourfold seemed just too long and the analogy too vague. At the time I was supposed to pass a habilitation exam, a kind of second, more advanced thesis, which qualifies one to apply for a full professorship. So I asked Claire whether she considered the current state of my research sufficient for that. She had a quick look at my files and then said exactly what I feared: "Why don't you just go on and prove potential density for those varieties with a self-map?" With Claire as a collaborator, this was indeed feasible. She could make the analogy precise and came up with intricate constructions relating, via the Chow groups and $l$-adic Abel-Jacobi invariants, some Terasomatype conditions on certain cohomology groups of certain subvarieties with the relevant geometric questions, such as the preperiodicity of those subvarieties. I only had to fill in the geometry of our particular manifolds, on which I had already been working for a long time.

In this essay I deliberately have not recalled Claire's most famous and significant work, since others are doubtlessly going to do it and I wanted my contribution to be more personal. Shortly after our collaboration another personal story began for me with the birth of my own children. For the reason mentioned in the beginning, I was not sure about the effect this would have on my research. But I have been very lucky with my family, and 
probably I have got enough inspiration from Claire to overcome such concerns.

\section{Olivier Debarre}

\section{Intense, Brilliant, Formidable}

I have known Claire Voisin for a very long time, close to forty years. I gave her oral examinations when she was a first-year student in 1979. She then entered the "École normale supérieure de jeunes filles" in 1981 (at that time, men and women went to different écoles normales in France). Daniel Perrin, who was teaching at that school at the time, told me that from the outset she stood out from other students.

Although she has considerably mellowed over the years, she was very intense and demanding in her initial engagements in the world of mathematics. While preparing her $\mathrm{PhD}$ thesis, she was appointed teaching assistant for a course taught by Luc Illusie. The story goes that she quickly decided that the material given in class was not sufficiently challenging and she turned her exercise sessions into a parallel, much faster course, to the dismay of most of the students. After this experience, she turned her talents more directly to research, obtaining a pure research position at the Centre National de la Recherche Scientifique.

\begin{tabular}{c}
\hline Her \\
students \\
were in awe \\
of her.
\end{tabular}

Claire has always been a very quick thinker, and when it came to mathematics she had little patience with details and people whose thinking was slower than hers. Her first article, published in Inventiones in 1986 and extracted from her $\mathrm{PhD}$ thesis, where she proved the Torelli theorem for cubic hypersurfaces of dimension 4 , contains the following warning (my translation): "The symbol (box) means: the proof is obvious or uninteresting" (and these boxes are liberally used in that article). This capacity to think more quickly than others impressed all those around her. Her students were in awe of her. One of her former students, now a successful mathematician, once told me that he was so terrified the days before an appointment with her that he could not get any sleep.

Mathematics was always central to her life. When I asked her once what she was doing over the summer vacation, she explained that she liked hiking in the mountains, but her husband (himself a very successful mathematician) liked the beach (or the other way around, I am not sure). Because of this divergence they chose to go to the countryside, so that both would find nothing else to do but work. But I wonder at times whether this is not an image she likes to give to the world.

For Claire is much more than a mathematician. She is the mother of four girls and one boy. After she gave

Olivier Debarre is professor in département de mathématiques et applications, École Normale Supérieure, Paris. His email address is olivier.debarre@ens.fr.

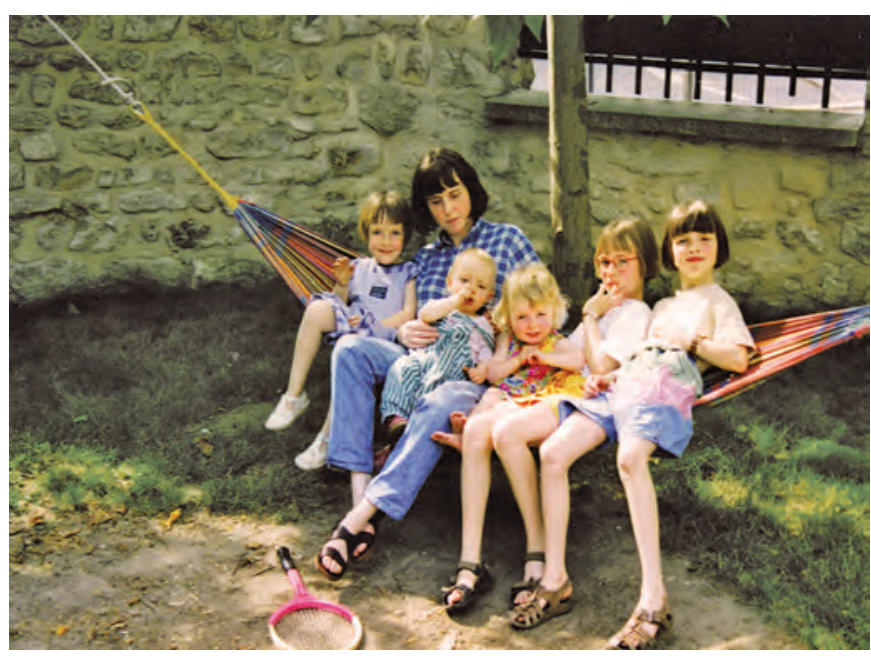

Voisin with her children in her garden (September 1997).

birth to her first daughter, she almost immediately left alone for a research visit to the University of Utah. When her colleagues learned about the situation, they quickly convinced her to go back to her baby! Children and music dominate her family life. Everybody in her family plays a musical instrument, although it seems that her musical ear does not help with foreign languages: once, at a seminar, she asked a question (in English) to the speaker, to which he responded: "I'm sorry, I don't understand French." She is also an enthusiastic wine lover; at a dinner party given after she gave a seminar talk, she warned, looking over the wine list, that she liked "very expensive wines."

Over the years, as she has gone from success to success, she has become a little less driven and more helpful when addressing others. She is now an excellent speaker. Her courses, although still relatively fast-paced, are very successful with students, and she has written a very popular reference book on Hodge theory based on these courses. She is very generous with her time (with students as well as with colleagues), and I believe that her students are no longer terrified of meeting with her. But her enthusiasm for mathematics is intact, and she is still remarkably intense.

It was only late in our careers that I actually had the chance to work with her. Claire had realized that a geometric construction of Peskine gave rise to a 20dimensional family of varieties of dimension 4 with trivial canonical bundle. She was convinced that these varieties were hyperkähler and suggested that we work together on this problem (we were both at the time visiting MSRI). I took the lazy way out and computed (with the help of the computer program Macaulay2, whose developers Daniel Grayson and Michael Stillman happened also to be at MSRI) their holomorphic Euler characteristic; this, by classification results, was enough to conclude. But Claire was not satisfied with this result and wanted to construct explicitly the nondegenerate holomorphic 2-form, which she did with Hodge theoretic 
arguments. Then there was the problem of proving that these varieties were deformations of Hilbert squares of K3 surfaces. Natural degenerations did not seem to work, so we had to find a round-about way. I will spare the reader the technical details, but we did in the end find a (not very elegant) solution, mainly thanks to Claire's obstination and technical powers. This was really when I realized that no difficulties were deemed by her too hard to overcome and that she would not stop until they had all been resolved.

This result is not one of Claire's main contributions to mathematics, but it illustrates her way of attacking a problem: once she gets an idea, she applies her formidable technical powers like a bulldozer and few obstacles resist her. In our case, she did not really care about the elegance of the proofs, eager as she was to move on to something else.

I had another occasion to collaborate with her (and my former student Zhi Jiang)

\section{Hodge theory is Claire's playground.}

on cohomology

classes whose pushforward by a morphism vanishes. The question addressed in the article, asked by Tommaso de Fernex, was to characterize these classes (in homology or cohomology) in terms of classes of subvarieties that are contracted by the morphism. Claire quickly saw that our conjectures would have very strong consequences in terms of the generalized Hodge conjecture-not a good sign for the resolution of our conjectures.

Hodge theory is Claire's playground. She knows it inside out from the theoretical point of view of course, but she also has an extraordinary ability to apply it to very concrete examples-she would rather prove a concrete consequence of a difficult conjecture than its equivalence to another, equally difficult, conjecture. This intimate knowledge of Hodge theory (and more) was what enabled her to make recent striking advances on very old questions about (stable) rationality of varieties.

I would like to finish by commenting on Claire's attitude towards being a (very successful) woman doing mathematics. Although she claims not to be in favor of making things easier for women, it is obvious that she is a role model for many women in mathematics. Being so brilliant, she can disregard the opinions (and derogatory comments) of some of her male colleagues, but she at times underestimates the difficulties other female mathematicians experience in our very masculine profession. Fortunately she has those four daughters, who have a very hard act to follow. Perhaps not surprisingly they are pushing her to realize that she has an important role to play as mentor to other women and as reminder to us all that the "mind has no sex," as Poulain de la Barre stated back in 1673. But some minds, be they male or female, are particularly incisive and impressive in the mathematical world. Claire's mind is unquestionably one of these.

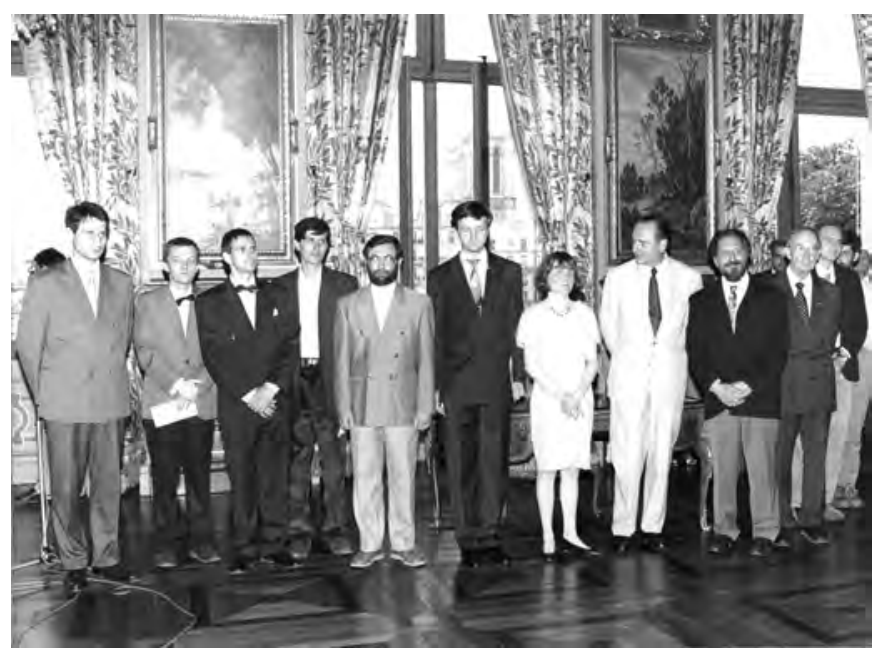

Voisin at Hôtel de Ville in Paris after the 1992 EMS prize. To Voisin's left are Jacques Chirac (then mayor of Paris, later president of France), Karoubi, and Hirzebruch. To her right are Franke, Goncharov, Kontsevich, and other awardees.

\section{Phillip Griffiths}

\section{The Leader in the Field of Complex Algebraic Geometry}

Over the past quarter century, Claire Voisin has been the leader in the field of complex algebraic geometry. In her work she has both solved many deep, long-standing geometric problems and established a number of results that delineate the field. Of particular personal interest is the extraordinary depth and ingenuity of her use of Hodge theory blended with the panoply of modern algebro-geometric methods. Although Claire has complete mastery of Hodge the-
She is a problem solver in the best sense of the word. ory and of the more formal algebraic and homological techniques in algebraic geometry, rather than add to these theories for their own sake her work enriches them by their use in solving difficult questions; she is a problem solver in the best sense of the word rather than a theory builder.

I will describe a number of ways in which Claire's work has delineated the field of complex algebraic geometry. As one example she showed that the naive interpretation of the Hodge conjecture for Kähler manifolds is false. To me this suggests that the purely complex analytic approaches such as the Kodaira-Spencer proof of the Hodge conjecture in codimension one (the Lefschetz

Phillip Griffiths is professor emeritus of mathematics at the Institute for Advanced Study. His email address is pg@math . ias.edu. 


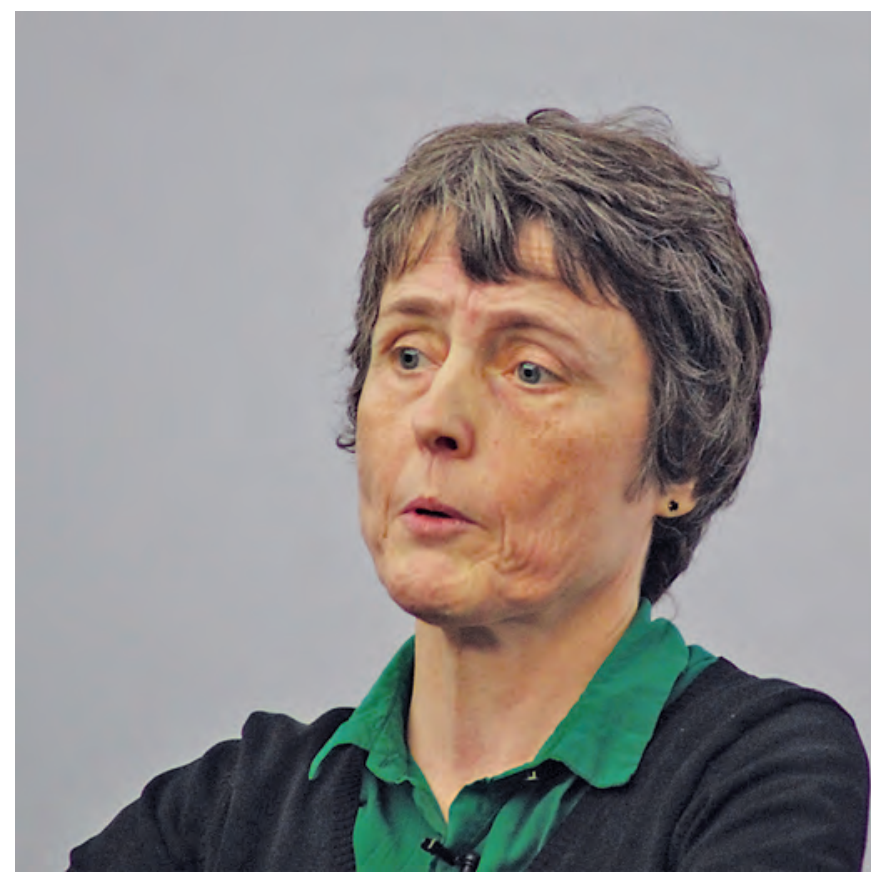

Voisin at the British Mathematical Colloquium 2014, Queen Mary University, London.

theorem) are unlikely to work. Some deeper and yet to be understood arithmetic aspects of the issue seem needed to solve the problem.

As a quite different sort of example of the way in which Claire's work greatly extends the frontiers of even the classical aspects of the field of algebraic geometry, there is nothing more basic than the structure of the equations that define a general algebraic curve. Here there is a central, deep conjecture due to Mark Green essentially stating that the syzygies of a general curve have the maximally harmonious structure. Her result that this holds for curves of odd genus went far beyond what was known and remains the best that we know today. This is one illustration of Claire's ability to completely settle, or at least greatly extend, what is known about the most interesting and challenging questions in the field.

\section{impeccable taste and judgment}

As another illustration of how Claire's work defines the boundaries of the field, it is well known that the topology of compact Kähler manifolds, and therefore of smooth projective varieties, has very special properties. A long-standing question was: Is every compact Kähler manifold $X$ homotopy equivalent to a smooth projective variety? For $\operatorname{dim} X=2$ the answer is affirmative (Kodaira, 1954). Claire gives examples to show that for $\operatorname{dim} X \geq 4$ there are $X$ 's that are not homotopy equivalent to a smooth projective variety. Variants of the question dealing with the bi-meromorphic equivalence class of $X$ are also shown to have negative answers.
Although I have not had the pleasure of collaborating directly with her, our paths cross often at conferences, and we met frequently over the 2014-15 academic year when she was a Distinguished Visiting Professor here at the Institute for Advanced Study. She is never without new insights and interesting ideas to discuss. I find it especially rewarding personally to follow her research, some of which builds on work I had thought about with my own students some four decades ago. I have learned a great deal from her, and I am proud to consider her both protégé and teacher.

Claire's work shows impeccable taste and judgment in the questions she chooses to address. They are central issues in the field, yet with her great ingenuity and skill they become accessible. Her work also has a "nononsense" character: here is an important and interesting question, one whose solutions will significantly extend our knowledge, and here without unnecessary frills and bells and whistles is a crisp and elegant solution of the problem.

\section{Daniel Huybrechts}

\section{A Passion for Geometry}

Whenever invited to speak at a conference with Claire Voisin in the audience, I am particularly nervous, and I like to think that I am not the only one. It is likely that at the end of a talk, especially on one of her numerous favorite topics, she understands the matter better than anyone else in the room, speaker included. She is likely to point out a crucial subtlety you have totally missed or may come up with a cleverer argument or a stronger result. At least, that is what happened to me. In any case, her questions are always dead-on target and likely to keep you thinking for some time. It seems almost impossible to discover a geometric construction involving Hodge theory that she has not thought of before or will not be able to enhance right away. Her technical prowess and her geometric intuition are mind-blowing and often, I find, a little intimidating.

Claire Voisin continues the famous French school of algebraic geometry, which traditionally has a distinctive arithmetic inclination, from a complex geometric perspective and is today the world's leading expert in Hodge theory as initiated by Pierre Deligne and Phillip Griffiths. Her choice of Arnaud Beauville as her PhD advisor was an early sign of her deep and lasting interest in the topology of algebraic varieties.

It is probably fair to describe the vast majority of her results as, in one way or the other, related to the Hodge conjecture. This Clay Millennium Problem attempts to describe the "visible part" of the geometry of a complex algebraic variety $X$ in terms of its classical topology encoded by its singular cohomology $H^{*}(X, \mathbb{Z})$ and its complex analytic structure reflected by the decomposition of differential forms according to their holomorphic and

Daniel Huybrechts is professor of mathematics at the Mathematical Institute of Bonn University. His email address is huybrech@math.uni-bonn.de. 
anti-holomorphic part $\oplus H^{p, q}(X)$. For example, Voisin's very first paper, resulting from her thesis, proves the Torelli theorem for hypersurfaces of dimension four described by one cubic equation such as $x_{0}^{3}+\cdots+x_{5}^{3}=0$. The theorem generalizes the classical result for Riemannian surfaces and asserts that such a variety is itself uniquely determined by its Hodge structure. She later proved Torelli theorems for further types of varieties like three-dimensional quintic hypersurfaces, which are of particular importance in mirror symmetry, another of her interests. Other papers of Voisin's explore various aspects of the Hodge conjecture itself, e.g., reducing the Hodge conjecture (for absolute Hodge classes) to the case of varieties defined over number fields or testing the original integral version of the conjecture.

From the very start, Voisin has also been interested in the "cohomologically invisible" aspects of the geometry of algebraic varieties encoded by the deeper layers of their Chow groups $\mathrm{CH}^{*}(X)$. According to a whole net of conjectures due to Bloch, Beilinson, Murre, Tate, and others, those cohomologically trivial slices should also be governed by cohomology, but by its nonalgebraic part. One of Bloch's conjectures predicts that the natural cycle map from the algebro-geometric $\mathrm{CH}^{*}(X)$ to the topological $H^{*}(X)$ is an isomorphism whenever it is surjective. Bloch's conjecture is inaccessible using the existing techniques and seems to be at least as difficult as the Hodge conjecture itself. The verification of any of these conjectures in geometrically meaningful examples is of great importance for the further flourishing of our field. And here, Voisin is at her best. Again and again, she has conceived some intricate geometric construction, often involving finite group actions, the yoga of diagonals, or higher AbelJacobi maps, to prove one of Bloch's conjectures. Her followers then hurry to digest her findings and to feed on the new pieces of exciting mathematics.

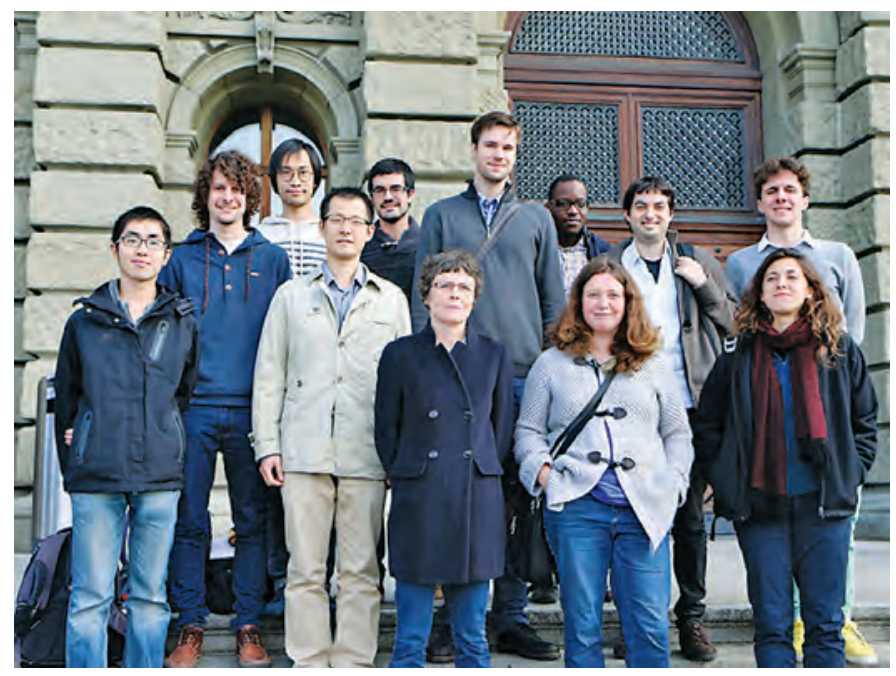

Here surrounded by some of her students and young algebraic geometers at the ETH at her 2015 Hopf Prize ceremony. Voisin is known for her generosity towards young mathematicians.
The theory of K3 surfaces, a class of surfaces particularly accessible to Hodge theoretic methods, is another of Voisin's favorite topics. She sheds new light on this special but geometrically extremely rich class of manifolds from various angles and often puts them to good use to approach a priori unrelated problems. For example, she successfully attacked Green's conjecture for algebraic curves by studying those that lie on a K3 surface. The conjecture itself deals with arbitrary algebraic curves and is meant to cast classical results of Noether and Petri about their canonical embeddings into a considerably enhanced framework. K3 surfaces are also central in Voisin's contributions to mirror symmetry and to cycle theoretic questions and in her investigation of hyperkähler manifolds.

Claire Voisin is gifted with a unique geometric insight. Again and again she has seen the potential of a particular construction or a certain point of view that eluded others. To name one recent example: Who would have thought that decomposing the diagonal, as a cycle or a cohomology class, with integral or rational coefficients would have any bearing on rationality questions like the famous Lüroth problem? The latter asks whether any subfield $L \subset \mathbb{C}\left(t_{1}, \ldots, t_{n}\right)$ of a purely transcendental field is purely transcendental. (The problem has an affirmative answer, algebraically and geometrically, in dimension one and, due to the classical Italian school of geometry, in dimension two.) Geometrically this is the question whether any unirational variety, one for which there exists a dominant algebraic map $\varphi: \mathbb{C}^{n} \rightarrow X$, is in fact rational. Due to the work of Clemens-Griffiths, Artin-Mumford, and Iskovskich-Manin the answer is known to be negative in dimension three and the easiest counterexamples are cubic and quartic hypersurfaces. This seems to settle the conjecture once and for all, but even more interesting than the conjecture itself have become the techniques developed for (dis)proving it (intermediate Jacobians, Brauer classes, birational rigidity, etc.). Voisin recently invented a subtler approach relying on the decomposition of the diagonal that has subsequently been improved and applied to many cases that were thought to be intractable.

Diagonals also come up in other and rather different contexts in Voisin's work. Let us look at just a few instances. According to Deligne, the Leray spectral sequence for a smooth projective morphism $f: X \rightarrow Y$ degenerates, and it does so on the level of complexes $R f_{*} \mathbb{Q} \cong \oplus R^{i} f_{*} \mathbb{Q}[-i]$. Just a few years ago Voisin asked the very natural question whether this can be realized multiplicatively. It cannot in general, but with her natural flair for combining topology and algebraic geometry, she uncovered a way to link the question to the decomposition of the diagonal and proved that a multiplicative splitting exists for families of K3 surfaces and other Calabi-Yau varieties, at least on a Zariski open (very large) part. 


\section{Her work on the so-called Kodaira problem is another example of her unbelievable geometric insight
and bravery.}

Diagonals also play a decisive role in her study of the Chow groups of K3 surfaces and hyperkähler manifolds. Her short paper with Beauville some ten years back, in which a distinguished zerocycle in the huge Chow group of any K3 surface was identified, and later papers on higher-

dimensional versions marked the dawn of a new association between Ricci-flat manifolds and algebraic cycles.

Her work on the so-called Kodaira problem is another example of her unbelievable geometric insight and bravery when it comes to settling long standing questions. By an ingenious construction she managed to detect tangible differences between general compact Kähler manifolds and those that can be described by polynomial equations. It turns out that there exist compact Kähler manifolds whose cohomology algebra $H^{*}(X)$ is not realized by any projective one and that therefore are topologically fundamentally different.

Despite being somewhat awe-inspiring, Voisin has become extremely popular among young algebraic geometers. She has supervised a number of excellent $\mathrm{PhD}$ theses and is known for her generosity towards her students and to young mathematicians in general. Her advanced courses on complex geometry and Hodge theory were crucial in the formation of a new generation of French complex geometers, and the English two-volume edition of her book on the subject has become the ultimate source for everyone wishing to learn about the modern theory of complex manifolds. Among my students, it has become an unspoken requirement and a good tradition to work through her book methodically. Voisin's deep love for algebraic geometry and her dedication to advance mathematics in general will continue to be an inspiration. The challenge is to keep up with her.

\section{François Charles}

\section{Variational Methods for Algebraic Cycles}

A major aspect of the work of Claire Voisin deals with applications of Hodge theory to algebraic cycles. Given a projective algebraic variety $X$-that is, the zero locus in complex projective space of a family of homogeneous polynomials - it is extremely hard in general to understand the topology of the various subvarieties of $X$, i.e., those submanifolds that can be cut out by homogeneous

François Charles is professor, Laboratoire de Mathématiques d'Orsay, Université Paris-Sud. His email address is francois .charles@math.u-psud.fr.

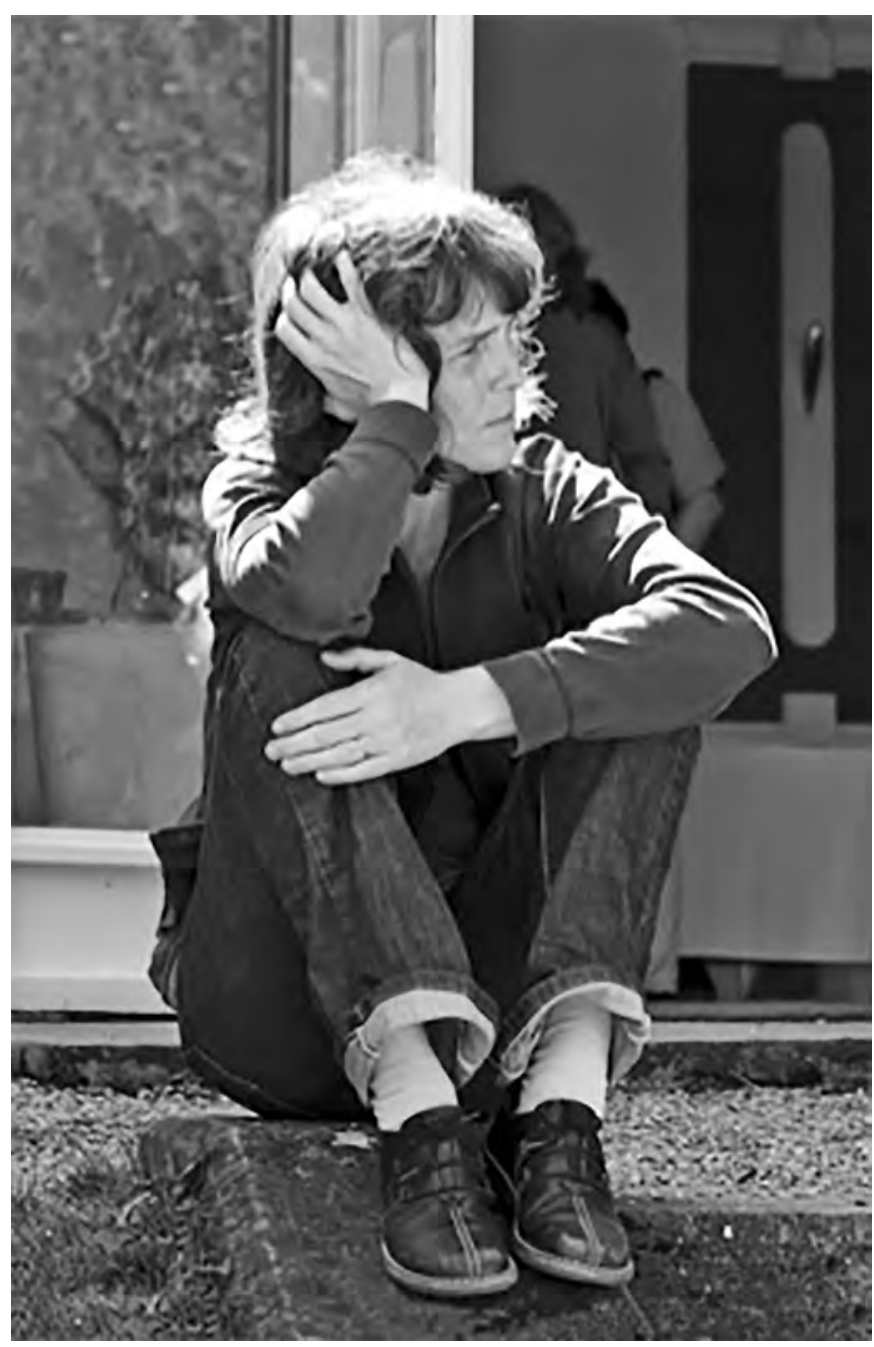

Voisin at IHÉS during the Simons Conference in September, 2007.

polynomials. Among others, the Hodge conjecture suggests the extent to which there exists a relationship between the topology of $X$, as reflected by its cohomology algebra, and the geometry of its subvarieties. We still have little understanding of how to construct subvarieties and how to understand equivalence relations between algebraic cycles-formal linear combinations of subvarieties.

Hodge theory provides a powerful set of invariants that reflect, on a cohomological level, the complex structure of $X$. The applications of these invariants to algebraic cycles predates in a way even Hodge theory itself and has been developed in the second half of the twentieth century by many mathematicians, most notably Griffiths and Deligne.

The work of Claire Voisin on algebraic cycles reflects a profound understanding of the implications, both geometric and topological, of deep conjectures on algebraic cycles to concrete statements on projective varieties. An important idea is the following: given a variety $X$ in com- 
plex projective space, one can consider the family of those subvarieties of $X$ cut out by hyperplanes. Hodge theory, through the notion of a variation of Hodge structures, provides a way to control the geometry of this family. This can be leveraged in two ways: we can use geometric insight on $X$ to understand the hyperplane sections, or we can use geometric insight on the hyperplane sections to understand $X$. The first idea has been applied by Voisin to the study of zero-cycles, formal linear combinations of points, up to rational equivalence, in the simple case where $X$ is the complex projective space. The following result is a clever application. It deals with a special case of the Bloch conjecture.

Theorem 1 ([Voi92], [Voi13]). Let $f$ be a homogeneous polynomial of degree $n$ in $X_{0}, \ldots, X_{n}$, invariant under the involution $i:\left(X_{0}, \ldots, X_{n}\right) \mapsto\left(-X_{0},-X_{1},-X_{2}, \ldots X_{n}\right)$. Then the action of $i$ on the Chow group of zero-cycles on the hypersurface defined by $f$ is trivial.

Chow groups of zero-cycles as above are enormous abelian groups built out of all points of the variety. They are notoriously hard to control, and they are expected to encode deep geometric information. The above theorem, which is one example of a more general result, is obtained through deep understanding of the constraints that Hodge theory, as in the work of Deligne, imposes on geometric objects.

The thorough understanding of how Hodge-theoretic structures manifest themselves in concrete cases is one of the most striking aspects of Claire Voisin's work, at least in my experience. Here is another example.

Theorem 2 ([Voi06]). Let $X$ be a uniruled or Calabi-Yau threefold. Then $X$ satisfies the integral Hodge conjecture.

The statement above means that any $\alpha \in H_{4}(X, \mathbb{Z})$, some multiple of which is cohomologous to an integral combination of curve classes, is itself cohomologous to an integral combination of curve classes. In the proof, it is the geometry of suitable hyperplane sections of $X$ that sheds light on $X$ itself: curves are simply found as curves on surfaces cut out by hyperplanes in $X$. Again, the most basic tool used to control the geometry of such families of surfaces had been developed before by Griffiths. However, using it in this setting requires geometric insight as well as technical mastery. It sheds light on the relationship between positivity properties of the canonical bundle and the integral Hodge conjecture, which does not hold in general.

This theorem has had considerable impact recently. It is a key input in rationality criteria for quadric bundles used by many authors. Recent work of Benoist shows that variants of this method can be used to prove de Jong's theorem on period-index for surfaces.

These two results and their proof reflect the deep influence of Claire Voisin's mathematics on me. She taught me, and shows in her papers, that far-reaching and difficult conjectures such as the Hodge conjecture have very concrete geometric consequences and that general motivic results can be leveraged against geometric insight in surprising ways.

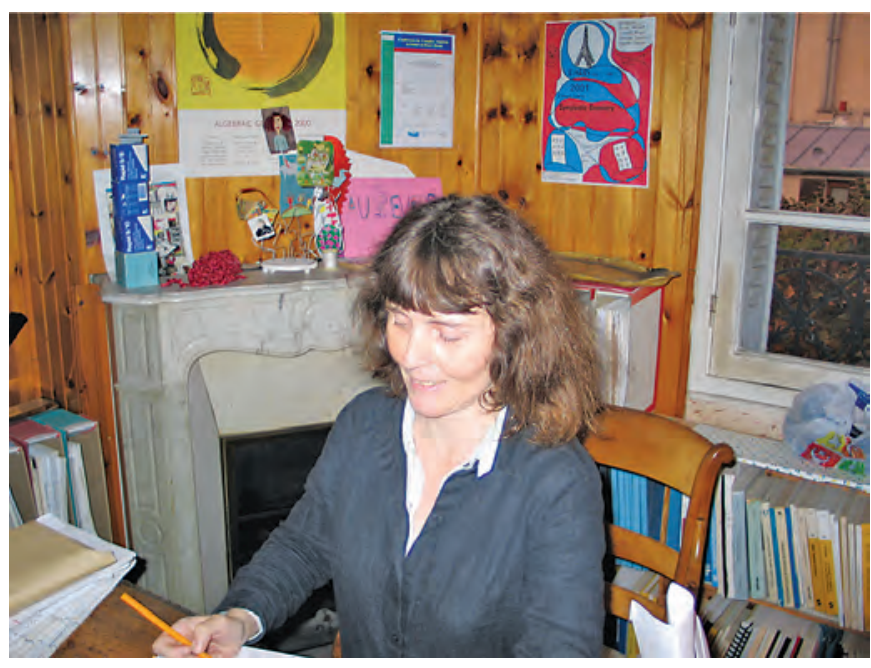

Voisin works in her office at home (2005).

The two results above come from a profound understanding of general properties of the locus of Hodge classes. Beyond their immediate consequences, I have myself been very influenced by this concrete incarnation of general principle-in particular, my first proof of the Tate conjecture for K3 surfaces stems from an application of related ideas to arithmetic geometry. Trying to understand the arithmetic analogues of the Hodge-theoretic principles above in specific situations has been an important aspect of my own work where Voisin's influence and geometric insight have been crucial, and at least from my perspective shows the wide impact of her mathematics.

\section{References}

[Voi92] Claire Voisin, Sur les zéro-cycles de certaines hypersurfaces munies d'un automorphisme, Ann. Scuola Norm. Sup. Pisa Cl. Sci. (4), 19(4):473-492, 1992. MR1205880

[Voi06] _ On integral Hodge classes on uniruled or CalabiYau threefolds. In Moduli Spaces and Arithmetic Geometry, volume 45 of Adv. Stud. Pure Math., Math. Soc. Japan, Tokyo, 2006, pp. 43-73. MR3099982

[Voi13] _ , The generalized Hodge and Bloch conjectures are equivalent for general complete intersections, Ann. Sci. Ec. Norm. Super. (4), 46(3):449-475 (2013), 2013.

\section{Photo Credits}

Opening photo courtesy of (CPatrick Imbert/Collège de France.

Photo of Claire Voisin with Peter Bühlmann (CDepartment of Mathematics, ETH Zurich.

Photos in Beauville, Debarre, Huybrechts, and Charles sections courtesy of Claire Voisin.

Photo of Voisin in Glasgow in the public domain.

Photo in Griffiths section courtesy of Bert Seghers. 